\title{
CORRESPONDENCIAS TONALES Y EL ORIGEN DE LOS CRIOLLOS INGLESES DEL ATLÁNTICO
}

\author{
Mario Portilla
}

\begin{abstract}
RESUMEN
El presente estudio tiene como finalidad ofrecer datos de tipo lingüístico que contribuyan a esclarecer la controversia acerca del lugar y el momento en que se gestó la variedad que dio origen a los criollos ingleses del Atlántico. El autor pretende mostrar que las correspondencias de ciertos patrones de distribución tonal encontradas en algunos criollos de base inglesa (especialmente entre el krio y el criollo inglés de Costa Rica) apoyan la hipótesis del origen americano del protocriollo ingles del Atlántico.
\end{abstract}

\begin{abstract}
The purpose of this study is to shed light on the controversy that exists about the place and time in which the language variety that originated English-based Atlantic creoles appeared. The author intends to prove, with linguistic data, that the correspondences found in certain tone distribution patterns (especially between Krio and the English Creole of Costa Rica) support the theory of an American origin for the English-based Atlantic Protocreole.
\end{abstract}

\section{Introducción}

\subsection{Hipótesis sobre el origen de los criollos ingleses del Atlántico}

El término criollos ingleses del Atlántico' fue introducido por Hancock (1969) para designar las variedades criollas de léxico inglés existentes tanto en Norte, Centro y Sudamérica, como en África Occidental, bajo la hipótesis de que estas variedades representan los descendientes modernos de un pidgin primitivo hablado probablemente a lo largo de la costa de África Occidental desde principios del siglo dieciséis.

Las claras similitudes e ineludibles correspondencias que existen a nivel fonológico, morfosintáctico y léxico entre los diversos criollos de base inglesa de ambos lados del Atlántico ha permitido postular un origen común para todas las variedades (cf. Hancock 1969 y 1987 inter alia).

Sobre este punto parece existir un consenso. Sin embargo, acerca de dónde y cuándo fue gestada la posible variedad original no lo hay. Dos posibles hipótesis han sido contempladas.

Una primera hipótesis, propuesta por Hancock $(1980,1986,1987)$, sugiere que todas estas variedades criollas provienen de una variedad criollizada de inglés surgida en la costa oc- 
cidental de África, problablemente a lo largo de la costa septentrional de Guinea hacia finales del siglo XVI y principios del XVII, la cual fue luego repidginizada y transportada a América durante la trata de esclavos en los siglos XVII y XVIII.

Hancock ha sugerido la existencia de variedades criollizadas de inglés en la costa de Guinea Septentrional ya desde principios del siglo XVII, producto de uniones entre los colonizadores europeos y la población nativa.

Cuando fueron establecidos fuertes para la trata de esclavos en la zona, la variedad de inglés utilizada en la interacción entre las primeras generaciones de criollos y la corte de sirvientes nativos hablantes de lenguas africanas habría sido difundida entre los esclavos, quienes la transportarían luego a América.

I attempted to show that a range of creolized varieties if English became established in settlements along the Upper Guinea coast which originated in the early seventeenth century in the family situations growing out of the unions between English speakers and Africans, and later between their children and the Africans who joined these communities. I believe there is sufficient historiographical and linguistic evidence to indicate that African slaves awaiting shipment to the western hemisphere had ample time to acquire some knowledge of Creole from the grumettoes who tended them, and that this continued to serve them as a lingua franca even after their arrival in the Americas (Hancock 1986: 96).

Según este autor, las diferencias estructurales que presentan actualmente los criollos derivados de esta variedad primitiva que se originó en África Occidental, denominada por él criollo inglés de la Costa de Guinea ('Guinea Coast Creole English'), son el resultado de las diversas circunstancias sociales y demográficas en las que históricamente esta variedad se desarrolló.

\begin{abstract}
It was the interaction of these, i.e. Creole [Guinea Coast Creole English] and different African languages (on the one hand), or Creole and English and different African languages (on the other), in different proportions in different places, which gave rise to the forms of the various local dialects, although in each case the social and topographical circunstances, as well as the rate at which the emerging creole fed back into this ongoing contact situation which allowed this to proceed, more or less differed. In the Suriname case, the significantly smaller metropolitan English component resulted in the creoles developing there diverging further from 'core creole'; elsewhere, e.g. in North America, the opposite situation caused the outcome to converge with metropolitan English (Hancock 1986: 95-6).
\end{abstract}

Es necesario recalcar que según esta hipótesis, todos los criollos de base inglesa existentes en la Costa Occidental de África se derivan últimamente de este primitivo criollo inglés de la Costa de Guinea.

En una segunda hipótesis alternativa, Cassidy (1980) propone que la variedad básica a partir de la cual se desarrollaron las variedades criollas existentes en Surinam, Jamaica y Carolina del Sur fue gestada en el Caribe, concretamente en Barbados.

Este autor supone que, aunque tal vez alguna variedad pidginizada o incluso criollizada de inglés pudiera haber existido ya en África Occidental, la base de todos los subsecuentes criollos atlánticos es la variedad de inglés hablada por los esclavos en Barbados durante el segundo cuarto del siglo XVII.

Whatever it was earlier and whatever it became later, the kind of English spoken by the slaves in Barbados in the second quarter of the seventeenth century must have been to some degree the source of Sranan, Jamaican Creole, and Gullah. (It is not claimed that a fully developed or consistent creole existed, merely that a common core may be reasonably assumed.) (Cassidy 1980) 
Cassidy propone explicar las diferencias estructurales entre los distintos criollos como producto de los distintos desarrollos históricos y demográficos que tuvieron posteriormente las diferentes regiones donde se desenvolvieron las variedades lingüísticas.

Hancock (1980:29), por su parte, cuestiona la hipótesis de la misma posible existencia de an early, distinctively Barbadian pidgin or creole based on English. Este autor supone que el inglés de Barbados no fue nunca realmente criollizado, debido al alto porcentaje de hablantes nativos de inglés en la isla en el período inicial de formación de la colonia. Además, afirma que, si bien es cierto Barbados fue el centro más importante para la colonización inglesa de Norteamérica, Sudamérica y el Caribe, la isla solo habría servido de temporary entrepôt, enlace entre África y su destino final.

Según Hancock, estos hechos se reflejan lingüísticamente en el supuesto hecho de que actualmente la variedad de inglés utilizada en Barbados está más cercana estructuralmente al estándar que ninguna otra variedad criolla del Caribe.

Sin embargo, hay que señalar que esta última observación hecha por Hancock es solo válida si se piensa que la criollización solo puede darse en los casos donde haya aparecido un pidgin que fuera estructuralmente muy distinto de la lengua de superestrato.

Además, desde el punto de vista lingüístico, la variedad de Barbados presenta rasgos afines con otros criollos atlánticos a nivel morfosintáctico (cf. Roy 1986), lo cual reflejaría la relación genética entre esta variedad y las otras del Caribe.

Desde el punto de vista histórico, varios hechos cuestionarían el uso extendido, en África Occidental, de alguna variedad pidginizada de base inglesa entre los esclavos que fueron transportados a cualquier colonia en América antes de 1665, año en que se supone que fueron fundadas las primeras factorías de esclavos por los ingleses en Gambia y Sierra Leona (LePage \& DeCamp 1960: 28; Hancock 1980: 26).

En primer lugar, tal como fue apuntado por Cassidy (1980) y por el propio Hancock $(1980,1986)$, no existen referencias explícitas de la existencia de ninguna variedad pidginizada o criollizada de inglés en África Occidental sino hasta finales del siglo diecisiete (Hancock 1980: 26), prácticamente un siglo después de que la colonia en Barbados ya había sido establecida.

En segundo lugar, ni la existencia de uniones mixtas de ingleses radicados en África con la población nativa, de las cuales habría nacido la primera generación de mulatos criollos, ni el establecimiento de fuertes por parte de los ingleses en la costa occidental de África para la trata de esclavos supone necesariamente el uso de tal variedad como medio de comunicación.

Es sabido que, cuando los ingleses -lo mismo que los holandeses y los franceses- se interesaron en el negocio de la trata de esclavos, ya los portugueses tenían establecidos fuertes de embarque de esclavos en toda la zona del Golfo de Guinea y el uso de un pidgin portugués había sido establecido allí desde mediados del siglo XV, el cual pudo haber sido usado por los ingleses -y otros europeos- como lingua franca (cf. Hellinger 1985: 72-88).

De hecho, los principales fuertes y puertos de los ingleses fueron aquellos tomados de los portugueses, en los cuales debió haber existido una variedad pidginizada de base portuguesa (Cassidy 1980: 10-2).

Otro hecho histórico recalcable es que solamente hasta 1665 los ingleses establecieron factorías para la trata de esclavos en la zona. Durante el período inicial de la colonización, los ingleses se proveyeron de esclavos africanos a través de los holandeses, quienes poseían el monopolio de la trata de esclavos para el Caribe no hispánico (Hancock 1977: 101-2, 1980: 26 y ss.).

Por lo tanto, no habría por qué suponer que, antes de 1665 , los esclavos importados por los holandeses tuvieran algún conocimiento de inglés antes de su llegada a Barbados, por lo cual es lógico esperar que los primeros esclavos adquirieran el inglés en la isla, en el contacto con la población europea ya establecida allí. Según Niles (1980: 65 y ss.), los trabajadores por 
contrato (indetured servants), en su mayoría irlandeses, habrían jugado un papel preponderante en el establecimiento de una variedad de inglés, pidgnizada por lo menos parcialmente, entre la población de esclavos africanos.

Los primeros esclavos utilizarían esta variedad de inglés, la cual tendría una forma bastante cercana a la variedad usada por los blancos, como pidgin con las sucesivas oleadas de esclavos que arribarían posteriormente a la isla.

Versiones diferentes de este pidgin habrían sido transportadas a las diferentes colonias inglesas en donde fueron criollizadas, tomando diversas formas de acuerdo con las circustancias demográficas en las que se desenvolvieron, pero conservando los rasgos esenciales de la variedad original.

Así, pues, el autor coincide con la hipótesis fundamental de Cassidy (1980) de postular el origen de los criollos del Caribe de base inglesa en la variedad lingüística utilizada en Barbados durante el segundo cuarto del siglo XVII.

\subsection{Propósito de la investigación}

Como se ha visto, la discusión acerca del lugar y el momento en que se gestó la variedad que dio origen a los criollos ingleses del Atlántico se basa especialmente en consideraciones históricas. El presente estudio tiene como finalidad ofrecer datos de tipo lingüístico que contribuyan a esclarecer esta controversia. El autor pretende mostrar que las correspondencias de ciertos patrones de distribución tonal encontradas entre algunos criollos de base inglesa (especialmente entre el krio y el criollo inglés de Costa Rica) apoyan la hipótesis del origen americano del protocriollo inglés del Atlántico.

\section{Los suprasegmentales en los criollos ingleses del Atlántico}

Los suprasegmentales constituyen el campo menos estudiado de los criollos ingleses del Atlántico, y a la vez el más controversial y de más difícil interpretación.

Con el fin de establecer una comparación lo más homogénea posible, en el presente estudio se parte de la consideración de que las denominadas lenguas de acento tonal (pitchaccent languages) son un tipo de lenguas tonales, al igual que lo son las denominadas lenguas tonales (tone languages) convencionales ${ }^{3}$. Por lo tanto, los suprasegmentales de altura (pitch) con valor fonémico de una lengua de acento tonal serán tratados como tonos (tonemas) y no como acento.

Por otra parte, se considera que las denominadas lenguas acentuales (accent languages) se oponen a las lenguas tonales (de acento tonal y de tono) en presentar el acento (stress) como suprasegmental.

Debido al escaso número de descripciones y datos confiables de los suprasegmentales de los criollos ingleses del Atlántico, solamente han sido tomadas en cuenta las siguientes variedades: CI de Costa Rica, CI de Guyana, sranan, saramaca, djuka, krio y wescos ${ }^{4}$.

El saramaca, el djuka, el krio y el wescos han sido descritos como lenguas que se ajustan a la definición convencional de lengua tonal. Estas variedades presentan contrastes tonales fonémicos relativos a cada sílaba, sin restricciones en la asignación de patrones de distribución tonal en la palabra. 
Las variedades de Costa Rica y Guyana, por otro lado, pueden ser clasificadas como lenguas de acento tonal. A diferencia de las lenguas tonales convencionales, estas lenguas presentan contrastes tonales relativos a la palabra y no a la sílaba; por lo tanto, tienen restricciones en la asignación del tipo y número de tonos que pueden ocurrir en una palabra.

El sranan ha sido descrito como una lengua de acento dinámico.

Consideramos conveniente hacer a continuación una descripción individual de cada una de las variedades comparadas.

\subsection{Variedades de acento tonal}

\subsubsection{El CI de Costa Rica}

Esta variedad presenta dos tonos de nivel que tienen carácter fonémico, un tono alto / ^/ y un tono bajo / $\dashv /$ (no marcado gráficamente en la transcripción fonológica)

Por regla general, toda palabra contiene solamente un tono alto. Sin embargo, existe un reducido número de palabras monosilábicas (algunos pronombres, preposiciones, conjunciones y marcadores temporales y aspectuales) que presentan tono bajo.

Algunos ejemplos de contrastes tonales son los siguientes:

$\begin{array}{lll}\text { án } & / \dashv / & \text { mano (hand) } \\ \text { an } & / \dashv / & y \text { (and) } \\ \text { fá́da } & / \dashv \dashv / & \text { padre (father) } \\ \text { fa:dá } & / \dashv \dashv / & \text { cura (father) } \\ \text { tíela } & / \dashv \dashv / & \text { sastre (tailor) } \\ \text { tiëlá } & / \dashv \dashv / & \text { Taylor (Taylor) }\end{array}$

Además, existen en esta variedad tres tonos de contorno, cuya aparición está condicionada por el contexto.

Existe una regla de descenso para la posición final de un enunciado afirmativo y para la citación de palabras. Esta regla hace predecible la aparición de los dos tonos de contorno descendentes en esta posición.

En monosílabos, el tono bajo se realiza obligatoriamente como un tono bajo descendente $[\checkmark]$. El tono alto, por su parte, puede ser realizado optativamente como un tono alto-descendente $[y]$ o como un tono alto $[-1]$.

En palabras polisilábicas, el tono bajo de la última sílaba se realiza optativamente o bien como un tono fonéticamente bajo [ $\lrcorner$ ], o bien como un tono bajo descendente [ $\checkmark$ ]. El tono alto, por su parte, se realiza optativamente o bien como un tono alto [ $\dashv$ ], o bien como un tono alto descendente [ $y$ ].

Por otro lado, las vocales largas condicionan la aparición de un tono de contorno ascendente. En esta variedad, las vocales largas se componen fonéticamente de dos segmentos o moras. Una vocal larga con tono bajo subyacente presenta ambas moras con tono bajo [\lrcorner$\dashv]$.

Pero una vocal larga con tono subyacente alto puede realizarse fonéticamente de dos formas diferentes: o bien ambas moras presentan un tono alto [ $\dashv \dashv]$, o bien la primera mora presenta un tono bajo y la segunda un tono ascendente [\lrcorner$\wedge]$.

Las palabras citadas anteriormente se realizan fonéticamente de la siguiente forma en posición final de enunciado: 


\begin{tabular}{|c|c|c|c|}
\hline án & [ án à ] & {$[\neg \sim y]$} & mano (hand) \\
\hline & [ ăn ] & {$[v]$} & $y($ and $)$ \\
\hline a & [ fáádà fàădà] & {$[\dashv \dashv ৩ \sim \dashv \Lambda \downarrow]$} & padre (father) \\
\hline i:đá & / t- / [ fààdá fààdā] & $[\dashv-\dashv \sim\lrcorner-y]$ & cura (father) \\
\hline
\end{tabular}

Además, existe en esta variedad una regla de asimilación tonal progresiva. Esta regla convierte todos los tonos bajos de un grupo fónico en tonos fonéticamente altos, cuando estos se encuentran después de un tono fonémicamente alto. Por ejemplo, véase la siguiente oración:

/ a sí mi fámılı / > [ à sí mí fámílí ( fámílì ) ]

$[\dashv \dashv \dashv \dashv \dashv>\dashv \dashv \dashv \dashv \dashv \dashv \sim \dashv \dashv ノ]$

Vi a mi familia (I saw my family)

\subsubsection{El CI de Guyana}

Diversos autores (Allsopp 1972; Carter 1983, 1987 y 1989 y Holder 1972) afirman que el criollo de Guyana es una lengua de acento tonal.

Según Carter (1987 y 1989), esta variedad presenta dos tonos fonémicos de nivel, un tono alto $\backslash \dashv \backslash$ y un tono bajo $\backslash \dashv \backslash$ (no marcado en la transcripción fonológica).

Toda palabra contiene solamente un tono alto. El tono (o acento tonal, en la terminología usada por Holder) tiene carácter léxico, por ejemplo 5 :

fá:da $/ \dashv \dashv /$ padre (father)

fa:dá / $/ \dashv /$ cura (father)

bátl / / batalla (battle)

batl $/ \dashv /$ botella (bottle)

Según Carter (1987: 23 y 1989: 35), esta variedad presenta una regla de descenso en oración o frase final afirmativa, por la cual un tono alto $\backslash \uparrow \backslash$ se convierte en un tono fonéticamente descendente $[\checkmark]^{6}$.

$\begin{array}{llll}\text { pa:tí } & / \dashv \dashv / & \text { [pà:tí] } & {[\dashv \mathrm{V}] \text { partido (party) }} \\ \text { akíunts } & / \dashv \dashv / & \text { [àkn̂unts] } & {[\dashv] \text { cuentas (accounts) }}\end{array}$

Además, esta variedad presenta también una regla de asimilación progresiva. En una palabra, todos los tonos fonémicos bajos que se encuentran después del tono alto fonémico se convierten en tonos fonéticamente altos. Por ejemplo, véase a continuación la siguiente frase:

/ junıvírsalı akséptabl altírnatıvz / > $\dashv\lrcorner \dashv\lrcorner\lrcorner \dashv-\dashv\lrcorner \dashv-\dashv\lrcorner$

[ jùnìvírsálí àkséptábị àltírnátívz ]

$\dashv \dashv \dashv \dashv \dashv \dashv ન \dashv-\dashv \dashv \dashv \dashv$ 


\subsection{Variedades tonales}

\subsubsection{El krio}

La mayoría de los autores (Berry 1961; Fyle \& Jones 1980; Carter 1989) concuerdan en que el krio presenta solamente dos tonos de nivel con carácter fonémico, un tono alto / $\dashv / \mathrm{y}$ un tono bajo / $\dashv /$ (no marcado gráficamente en las transcripciones fonológicas).

A diferencia de las variedades de acento tonal descritas anteriormente, el krio no presenta restricciones en la cantidad de tonos altos o tonos bajos que pueden aparecer en una palabra polisilábica, por lo cual se considera que esta variedad es una lengua de las llamadas tonales.

El tono tiene tanto carácter léxico como gramatical. Al igual que en el caso de Costa Rica, algunas preposiciones, pronombres, conjunciones y auxiliares verbales presentan consistentemente tono bajo.

Algunos ejemplos de contrastes tonales son los siguientes ${ }^{7}$ :

$\begin{array}{lll}\text { án } & / \dashv / & \text { mano (hand) } \\ \text { an } & / \dashv / & y \text { (and) } \\ \text { fáda } & / \dashv \dashv / & \text { padre (father) [satíricamente] } \\ \text { fađáá } & / \dashv \dashv / & \text { cura (father) } \\ \text { dé } & / \dashv / & \text { allá, dia (there, day) } \\ \text { de } & / \dashv / & \text { marcador de progresivo }\end{array}$

Carter (1989) ha señalado que en krio existe una regla de descenso en la posición final de enunciado afirmativo, por la cual un tono fonémicamente alto / $\dashv /$ se convierte en un tono fonéticamente descendente [ $\mathrm{V}$ ].

Según los datos ofrecidos por Fyle \& Jones (1980: 22), la regla de descenso parece estar condicionada por la estructura fonológica de la palabra y por su naturaleza semántica. Así, el tono alto será fonéticamente descendente en todos los monosílabos (excepto en aquellos que son nombres propios) y en toda palabra polisilábica que no termine en vocal, lateral o nasal.

\begin{tabular}{|c|c|c|c|c|}
\hline ḑ̧́n & $|\neg|$ & [đ̧3ón] & {$[-1]$} & John (John) \\
\hline 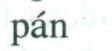 & $|\dashv|$ & [pân] & [ v & sartén (pan) \\
\hline & $\mid+-1 /$ & [wàtá] & {$[\dashv-1]$} & agua (water) \\
\hline & $|+-1|$ & [tròsîs] & {$[\dashv v]$} & pantalones (trousers) \\
\hline
\end{tabular}

Carter (1989) señala que en krio aparece una tendencia esporádica de asilimación tonal progresiva, por la cual un tono bajo que sigue a un tono alto se manifiesta como fonéticamente alto.

\subsubsection{El wescos}

Carter (1987 y 1989) y Criper-Friedman (1990) proponen la existencia de dos tonos de nivel con carácter fonémico, un tono alto / $\dashv /$ y un tono bajo / $\lrcorner /$ para esta variedad.

Por su parte, Dwyer (n.d. [1967]) supone la existencia de tres tonos fonémicos de nivel, un tono bajo, un tono alto débil ( $a$ weak high tone) y un tono alto fuerte (a strong high tone).

Sin embargo, según los propios datos ofrecidos por el autor, se puede deducir que la diferencia entre los dos tonos altos está condicionada por la estructura fonológica de la palabra, al igual que sucede en el caso del krio. El tono alto fuerte ocurre principalmente en monosílabos. 
Además, en palabras polisilábicas aparece en posición final, cuando estas terminan en consonante. El tono alto débil ocurre en palabras polisilábicas en posición inicial, intermedia y en posición final solo cuando la palabra termina en vocal.

Ejemplos de las oposiciones tonales son los siguientes ${ }^{8}$ :

$\begin{array}{lll}\text { hán } & / \dashv / & \text { mano (hand) } \\ \text { an } & / \dashv / & \text { y(and) } \\ \text { bába } & / \dashv-1 & \text { barbero (barber) } \\ \text { babá } & / \dashv 1 / & \text { padre } \\ \text { dé } & / \dashv / & \text { día }(\text { day) } \\ \text { de } & / \dashv / & \text { marcador de progresivo }\end{array}$

Tanto Carter (1987 y 1989) como Criper-Friedman (1990) mencionan que el wescos presenta una regla de descenso en posición final de enunciado (cadencia final).

Según Criper-Friedman (1990), por efecto de esta regla de descenso, un tono bajo se realiza fonéticamente como un tono levemente descendente y un tono alto como un tono descendente.

Carter (1987 y 1989) menciona una regla de asimilación progresiva del tono alto, por la cual solamente el tono bajo de la sílaba siguiente a este se realiza como un tono fonéticamente alto.

Criper-Friedman (1990) muestra, sin embargo, que esta regla de asimilación progresiva se extiende a todas las sílabas de un grupo tonal. Véase el siguiente ejemplo?:

What a very unfortunate incident!

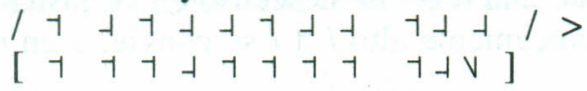

¡Qué incidente tan desafortunado!

\subsubsection{El saramaca}

Según Voorhoeve (1959) esta variedad presenta dos tonos de nivel con carácter fonémico, un tono alto / $\dashv /$ y un tono bajo / $\dashv /$ Al igual que en las variedades descritas de África Occidental, el saramaca no presenta restricciones en la distribución de tonos altos y bajos en una palabra.

Ejemplos de contrastes tonales son los siguientes ${ }^{10}$ :

$\begin{array}{lll}\text { tú } & / \dashv / & \operatorname{dos}(\text { two }) \\ \text { tu } & / \dashv / & \text { también (too) } \\ \text { maaká } & / \dashv-1 / & \text { notar (portugués marcar) } \\ \text { maáka } & / \dashv-1 / & \text { presagio } \\ \text { maká } & / \dashv-/ & \text { espina (macaw) }\end{array}$

Rountree (1972) señala que el saramaca presenta una regla general de descenso en posición final de enunciado. En enunciados afirmativos no enfáticos, el tono alto de la última sílaba de la palabra se realiza como un tono fonéticamente medio [ $\dashv$ ] y el tono bajo se realiza como un tono fonéticamente extra bajo [\lrcorner$]$. 


\section{/ dí káima kulé alá / > [ dí káimà kùlé àlā ]

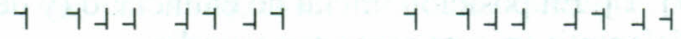 \\ El lagarto corre allá
/ mi tá wáka / > [ mì tá wákà ]

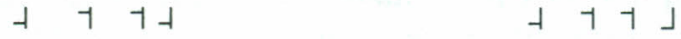 \\ Estoy caminando}

Según Voorhoeve (1961) y Rountree (1972), esta variedad presenta una regla general de asimilación progresiva. Por esta regla, todos los tonos fonémicamente bajos de un determinado sintagma que están tras un tono alto se realizan fonéticamente como tonos altos. Según estos autores, la aplicación de esta regla depende de la clase de palabras que componen el sintagma. Rountree menciona cuatro distintas clases de palabras en saramaca, dos de las cuales permiten la aplicación de la regla y dos que la opacan (cf. Rountree 1972: 314 y ss.). Véanse los siguientes ejemplos.



\subsubsection{El djuka}

Según Huttar \& Huttar (1972), el djuka presenta dos tonos de nivel con carácter fonémico, un tono alto $/ \dashv /$ y un tono bajo $/ \dashv /$.

Ejemplos de contrastes tonales son los siguientes ${ }^{11}$ :

$\begin{array}{lll}\text { búkú } & / \dashv \dashv / & \text { libro (book) } \\ \text { buku } & / \dashv-/ & \text { hongo } \\ \text { bákáá } & / \dashv \dashv-/ & \text { hombre blanco } \\ \text { baáká } & / \dashv \dashv-/ & \text { negro (black) }\end{array}$

De acuerdo con estos autores, tal como se nota en los ejemplos anteriores, en esta lengua no existen palabras que presenten tonos bajos después de un tono alto. Esta restricción podría ser interpretada quizá como el producto de una regla general de asimilación progresiva de los tonos altos. Por esta regla, esta variedad se asemejaría al tipo de lenguas de acento tonal descritas anteriormente. 
En posición final de enunciado (ante una pausa final), el tono alto de la última sílaba se realiza como un tono fonéticamente medio [ $\dashv$ ] . En posición inicial de enunciado (y después de pausa), el tono alto también puede realizarse optativamente como tono medio.

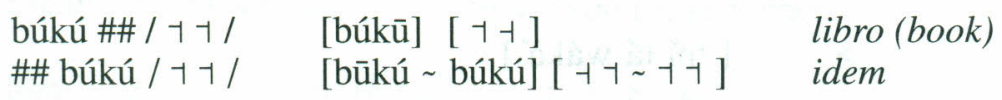

En posición final de enunciado, el tono bajo de la última sílaba de una palabra que presenta solamente tonos bajos se realiza como un tono fonéticamente alto.

$$
\text { buku \#\#/ } / \dashv / \quad \text { [bùkú] [ }
$$

Ambas reglas pueden ser interpretadas como variantes de una misma regla general de disimilación que opera en djuka.

\subsection{Variedades con acento dinámico}

\subsubsection{El sranan}

Según Echteld (1961), el sranan es una lengua de acento dinámico. En esta lengua, el acento es un rasgo exclusivo de intensidad (stress), en la cual la altura no parece ser un rasgo fonético notable. El acento tiene, además, un carácter contrastivo ${ }^{12}$.

'buku libro (book)
bu'ku moho
'pina alfiler (pin)
pi'na indigente
mativos.

El sranan presenta, además, un descenso en altura en posición final de enunciados afir-

\section{Las correpondencias tonales}

De las variedades mencionadas solamente el sranan ha sido descrito como una lengua eminentemente de acento dinámico. El CI de Costa Rica y el CI de Guyana pueden ser clasificados como lenguas de acento tonal. Presentan patrones de asignación y de realización de los tonos que son muy semejantes entre sí. El saramaca, djuka, krio y wescos se presentan como lenguas tonales convencionales. El krio y el wescos, por su parte, presentan también muchas semejanzas en cuanto a la realización de los tonos, entre sí y con respecto de los criollos de Costa Rica y Guyana.

Tanto las lenguas de acento tonal como las tonales presentan dos tonos de nivel con carácter fonémico: un tono alto / ' / y un tono bajo / / El sranan presenta, por supuesto, solamente una sílaba acentuada por palabra.

Existen varios indicios que permiten suponer que la protovariedad de la cual provienen los criollos ingleses del Atlántico era originalmente o bien una lengua de acento tonal (como el 
CI de Costa Rica y el CI de Guyana), o bien una lengua de acento dinámico (como el sranan). En primer lugar, los cognados existentes que son comunes para todas las variedades presentan solamente un tono alto (o acento en sranan) por palabra polisilábica ${ }^{13}$. En segundo lugar, tampoco existen cognados de las palabras polisilábicas con tonos bajos solamente. Existe un reducido número de palabras monosilábicas con tono bajo, sin embargo, se trata de palabras de carácter gramatical (pronombres, conjunciones, marcadores de tiempo y aspecto), las cuales son comúnmente inacentuadas en lenguas de acento dinámico.

A continuación se presenta una lista de algunos cognados de las variedades comparadas ${ }^{14}$.

$\begin{array}{lllllll}\text { SRA } & \text { SAR } & \text { DJU } & \text { KRI } & \text { WES } & \text { COS } & \\ \text { 'dron } & \text { doón } & \text { doón } & \text { dróm } & \text { dróm } & \text { drím } & \text { tambor (drum) } \\ \text { 'liba } & - & \text { líba } & \text { ríva } & - & \text { ríva } & \text { río (river) } \\ \text { 'ebi } & \text { ébi } & \text { ébi } & \text { ébi } & \text { hévi } & \text { Évi } & \text { pesado (heavy) } \\ \text { si'don } & \text { sindó } & \text { sidón } & \text { sidóm } & \text { sidón } & \text { sidín } & \text { sentarse (sit down) } \\ \text { ma'ka } & \text { maká } & \text { !máka's } & - & - & \text { maká } & \text { espina (macaw) } \\ \text { bi'kasi } & \text { biká } & \text { bikáa } & \text { bikós } & - & \text { biká:s } & \text { porque (because) } \\ \text { ta'baka } & - & \text { tabáka } & \text { tabáka } & \text { tabáka } & \text { tabáka } & \text { tabaco (tobacco) } \\ \text { mas'kita } & - & \text { makisíta } & \text { maskíta } & \text { moskíto maskíta } & \text { mosquito (mosquito) }\end{array}$

En los ejemplos anteriores se nota que existe en todas las variedades una correspondencia en la asignación de lugar del tono (o acento) en la palabra. Es importante señalar que, en estos casos, el tono alto (o acento en sranan) corresponde al acento y el tono bajo (o ausencia de acento en sranan) a la ausencia de acento en inglés estándar.

Sin embargo, existe un cierto número de palabras que no presenta esta correspondencia en la asignación del lugar del tono, tal como se observa en la siguiente lista de cognados:

$\begin{array}{llllllll}\text { SRA } & \text { SAR } & \text { DJU } & \text { KRI } & \text { WES } & \text { COS } & \text { GUY } & \\ \text { 'bobi } & \text { bóbi } & \text { bóbi } & \text { bobí } & \text { bobí } & \text { b } \Lambda \text { bí } & - & \text { teta (bubby) } \\ \text { 'doti } & - & \text { dóti } & \text { dotí } & \text { dotí } & \text { d } \Lambda(\mathrm{r}) \text { tí } & \text { d } \Lambda \text { rtí } & \text { polvo, sucio (dirty) } \\ \text { 'watra } & \text { wáta } & \text { watáa } & \text { watá } & \text { watá } & \text { wartá } & \text { wa:tá } & \text { agua (water) }\end{array}$

De acuerdo con los ejemplos anteriores, los criollos de Surinam han conservado el lugar de asignación del tono (o acento para el sranan) que originalmente existía en inglés para el acento, mientras que las demás variedades han sufrido una innovación.

El número de palabras que han sufrido la innovación y que, por tanto, presentan diferencias en la asignación del lugar del tono alto en relación con el acento en inglés es considerable. A continuación se ofrece una lista más amplia de estas palabras en krio, CI de Costa Rica y sranan, con el fin de tratar de ilustrar en qué consiste la innovación y de determinar su posible condicionamiento ${ }^{16}$.

\begin{tabular}{|c|c|c|c|}
\hline $\begin{array}{l}\text { KRI } \\
\text { Ja }\end{array}$ & $\cos$ & SRA & \\
\hline pusí & pusí & pus'pusi & gatito (pussy) $^{17}$ \\
\hline monkí & $\mathrm{m} \wedge \mathrm{jkí}$ & 'monki-'monki & mono (monkey) \\
\hline moní & m^ní & 'moni & dinero (money) \\
\hline graní & graní & 'grani & abuela (granny) ${ }^{18}$ \\
\hline torí & (s)tuorí & 'tori & cuento (story) \\
\hline
\end{tabular}




\section{Ib}

Ébi

twénti

plénti

Éni

IIa

kokó

natái

knkí

natí

'hebi

'twenti

'eni

pesado (heavy)

veinte (twenty)

mucho (plenty)

cualquier (any)

cacao (cocoa)

achiote (nut oil)

IIb

jála

náro

jélı

nárı

'jala

amarillo (yellow)

estrecho (narrow)

\section{IIIa}

gwevá

fadá

watá

gwa:vá

fa:dá

wa:tá

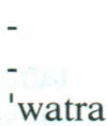

guayaba (guava)

sacerdote, padre (father)

hígado (liver)

IIIb

líba

fája

pépe

fáda

óda

líva

fája

pépa

fá:da

áda

'liba

'faja

'pepre

hígado (liver)

fuego (fuego)

pimienta (pepper)

padre (father)

otro (other)

Como se nota en la lista anterior, en el caso de palabras simples (es decir, no de compuestos), la innovación del cambio de lugar del tono alto está condicionada, por una parte, por la terminación vocálica de la palabra. Por otra parte, además las palabras deben ser bisilábicas.

Otra lista de palabras, que se presenta a continuación, muestra que el cambio no ocurrió cuando la palabra termina en consonante (grupo IV) ni cuando la palabra tiene más de dos sílabas (grupo V).

\begin{tabular}{|c|c|c|c|}
\hline \multicolumn{4}{|l|}{$\begin{array}{l}\text { KRI } \\
\text { IV }\end{array}$} \\
\hline bótul & bákl & 'batra & botella (woman) \\
\hline pádul & págl & 'pari & remo (paddle) \\
\hline pípul & píspl & 'pipel & gente (people) \\
\hline úman & úman & 'uma & mujer (woman) \\
\hline rótin & rátın & - & podrido (rotten) \\
\hline sévin & sévin & 'seibi & siete (seven) \\
\hline ózban & ńzban & - & esposo (husband) \\
\hline óndred & índred & - & cien (hundred) \\
\hline 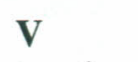 & & & \\
\hline tumára & tumára & 'tamara & mañana (tomorrow) \\
\hline  & pitíeta & pa'tata & papa (potato) \\
\hline ta'baka & tabáka & tabáka & tabaco (tobacco) \\
\hline
\end{tabular}




$\begin{array}{llll}\text { mas'kita } & \text { maskíta } & \text { maskíta } & \text { mosquito (mosquito) } \\ \text { dzeméka } & \text { dzứŕeka } & - & \text { Jamaica (Jamaica) } \\ \text { aligéta } & \text { aligéta } & - & \text { lagarto (alligator) }\end{array}$

Otro aspecto por destacar es el hecho de que la innovación mencionada solamente ocurrió en palabras sustantivas (grupos Ia y IIa) y no en modificadores (pronombres, numerales y adjetivos) (grupos Ib y IIb).

La mayor parte de los rubros del grupo III se trata de palabras que presentaban, en posición final, una consonante / -r / en inglés moderno temprano. Esta puede ser la razón por la cual en casi todos estos casos no ocurriera la innovación (grupo IIIb). Sin embargo, en algunas pocas ocasiones, a pesar de que presentan también una -r final ortográfica, los rubros correspondientes a agua y sacerdote exhiben la innovación mencionada. El uso frecuente de la palabra sacerdote como modificador puede haber motivado el cambio de lugar del tono.

Además, en el CI de Costa Rica, al igual que en el CI de Guyana, se pueden agregar unos dobletes correspondientes a apellidos y nombres comunes, en los cuales el nombre propio presenta el cambio de lugar del tono alto (a pesar de tratarse de palabras que presentan una -r final ortográfica), mientras que el nombre común conserva el lugar original del tono alto; por ejemplo, / tiela! / Taylor (< Taylor) : / tíela / sastre (< tailor). El uso extensivo de los nombres propios como vocativos pudo haber propiciado el cambio tonal (cf. Berry 1976: 269, para el caso del CI de Jamaica).

Por último, la innovación parece haberse efectuado en palabras o bien que son compuestos o bien de verbos que presentaban originalmente una partícula locativa.

\begin{tabular}{|c|c|c|c|}
\hline $\begin{array}{l}\text { KRI } \\
\text { VI }\end{array}$ & $\cos$ & SRA & \\
\hline sidóm & sidún & si'don & sentarse (sit down) \\
\hline tináp & (s)tanńp & ta'napu & levantarse (stand up) \\
\hline futból & futbá:l & - & fútbol (football) \\
\hline kok(o)nát & kıkanát & $\mathrm{k}(\mathrm{o})^{\prime}$ kronto & sentarse (sit down) ${ }^{19}$ \\
\hline fijamán & fIJamán & fisi'man & pescador (fisherman) ${ }^{20}$ \\
\hline
\end{tabular}

La lista anterior (grupo VI) muestra, sin embargo, además que la falta de correspondencia entre el lugar del acento en inglés estándar y el lugar del tono (o acento) es común a las tres lenguas criollas comparadas; es decir, incluye también al sranan. Esto puede indicar que quizá la variedad de inglés no estándar que dio origen al protocriollo inglés del Atlántico presentara originalmente, en estos casos, el acento en un lugar distinto del inglés estándar.

Por último, vale la pena recordar de nuevo que, en palabras bisilábicas agudas originalmente en inglés (grupo VII), las variedades comparadas coinciden en asignar un tono alto en el lugar que corresponde al acento en inglés. Esto demuestra que las lenguas criollas de Surinam han mantenido los patrones de asignación de los suprasegmentales igual que como eran originalmente en inglés.

$\begin{array}{llll}\text { KRI } & \text { COS } & \text { SRA } & \\ \text { VII } & & & \\ \text { bifó } & \text { bifúor } & \text { bi'fosi } & \text { antes (before) } \\ \text { bikós } & \text { brká:s } & \text { bi'kasi } & \text { porque (because) } \\ \text { tidé } & \text { tidíe } & \text { ti'de } & \text { hoy (today) } \\ \text { bigín } & \text { bigín } & \text { bi'gin } & \text { empezar (begin) } \\ - & \text { maká } & \text { ma'ka } & \text { espina (macaw) }{ }^{21}\end{array}$


Con palabras cognadas procedentes de lenguas africanas y del portugués (grupos VIIIa y VIIIb), las cuales probablemente habrían sido incorporadas al protocriollo inglés del Atlántico en época temprana, ocurre lo mismo que se ha mencionado para el caso de las palabras inglesas. Es decir, la innovación ocurre en el krio y en el CI de Costa Rica si la palabra presenta el contexto para ello (grupo VIIa). Las tres variedades comparadas conservan el lugar del tono alto en las palabras que no presentan en contexto para llevar a cabo la innovación.

\begin{tabular}{|c|c|c|c|}
\hline KRI & $\cos$ & SRA & \\
\hline- & nansí & a'nansi & araña (Twi ananse) 22 \\
\hline sabí & - & 'sabi & saber (Port. saber) \\
\hline \multicolumn{4}{|l|}{ VIIIb } \\
\hline pikín & pikní & pi'kin & niño (Port. pequenino) 23 \\
\hline pupú & pupús & pu'pu & heces (Twi popoe, Wolof pup) 24 \\
\hline potopóto & - & poto'poto & barro (Yoruba potopoto) \\
\hline aráta & - & a'lata & rata (?) \\
\hline- & bakrá & ba'kra & hombre blanco (Efik mbakara) \\
\hline únú & únv & 'unu & pronombre 3 pl. (Igbo unu) \\
\hline
\end{tabular}

\section{La cronología de la expansión inglesa en el Atlántico y el origen de la inno- vación fonológica}

Como se ha mencionado en la introducción, la controversia acerca del origen del protocriollo inglés del Atlántico se basa fundamentalmente en la disputa sobre el origen del krio. La hipótesis de Hancock (especialmente en 1986) de que esta variedad criolla proviene de un pidgin que se gestó en la costa meridional de Guinea hacia finales del siglo XVI y principios del siglo XVII representa, a la luz de los datos lingüísticos ofrecidos anteriormente, una cierta contradicción.

Esta consiste en que si pensamos que el krio ha heredado la innovación del cambio de lugar del tono alto con respecto del lugar del acento que originalmente presenta el inglés, entonces habría que considerar que otras variedades que también han heredado esta innovación lingüística en América tienen el mismo antepasado que el krio. Esto es, pues, lo que se supondría que sería el caso para el CI de Guyana y para el CI de Costa Rica.

Por otro lado, dado que, definitivamente, ninguno de los criollos de Surinam comparte la innovación con el krio, el CI de Guyana y el CI de Costa Rica y dado que el sranan, el saramaca y el djuka más bien siguen el mismo patrón suprasegmental del inglés (con excepción de las palabras compuestas), se debería pensar que los criollos ingleses de Surinam provienen de un antepasado diferente que aquel del krio, del CI de Guyana y del CI de Costa Rica. Sin embargo, esta suposición no es sostenible ni desde el punto de vista histórico (cf. Holm 1986) ni desde el punto de vista lingüístico (cf. Hancock 1969).

Históricamente, la primera colonia británica que se establece en el Caribe es St. Kitts en las Islas de Sotavento en 1624. Luego de fundar otras colonias en las actuales Islas de Barlovento Holandesas, los ingleses se establecen en Barbados en 1627. Pronto, esta isla se convierte en la colonia más próspera de la región y en el principal punto estratégico de expansión territorial de los ingleses. Barbados juega un papel preponderante en el establecimiento de las primeras colonias importantes en el Caribe durante el siglo XVII: Surinam (1651), Jamaica (1665), Carolina (1672) y Guyana (1740). 
En 1651 un grupo de 100 hombres procedentes de Barbados funda una colonia en Surinam. En 1661 hay establecidos allí 1.000 colonos ingleses con 2.000 esclavos africanos. En 1665 , el número se eleva a 1.500 y 3.000 respectivamente. En 1667, Surinam es cedida a Holanda por los ingleses por el Tratado de Paz de Breda. Entre 1671 y 1675, la mayoría de los colonos ingleses ya han abandonado la colonia. En 1701, la población de la Colonia se compone de 20 colonos ingleses, 700 colonos europeos no británicos y 7.500 esclavos africanos (cf. Voorhoeve 1970: 55-7). De esta forma, la presencia inglesa efectiva en Surinam dura apenas escasos veinticinco años hasta finales del siglo XVII y ocurre exclusivamente a través de Barbados. A partir del siglo XVIII, el contacto de los criollos de Surinam con el inglés es prácticamente nulo, por lo que cualquier influencia lingüística posterior de esta lengua sobre el sranan, el saramaca y el djuka queda completamente descartada.

En 1665, una expedición procedente de Barbados (con un contingente de hombres procedentes de la Islas de Barlovento) logra tomar la isla de Jamaica entonces en manos de los españoles. A partir de 1670, Jamaica se convierte en el punto de partida de la colonización del Caribe Occidental y de América Central. Desde Jamaica se fundan y eventualmente administran colonias en Belice (1670), Las Islas Caimán (1670), La Mosquitia de Nicaragua y Honduras (1675) y Providencia y San Andrés (1787). Durante el siglo XIX, una gran cantidad de trabajadores procedentes de Jamaica emigran a Panamá (1827) y a Costa Rica (1871) para trabajar sobre todo en la costrucción del Canal de Panamá y de vías ferroviarias.

Así, pues, según los datos históricos relatados es imposible negar el parentesco entre la variedad gestada en Barbados y las lenguas criollas de Surinam, el CI de Guyana, el CI de Jamaica y, por supuesto, también el CI de Costa Rica.

Por otra parte, lógicamente, si se insistiera en postular el origen africano del protocriollo inglés del Atlántico, existiría aún la posibilidad de pensar que tanto el krio como el CI de Costa Rica, por ejemplo, hubieran sufrido la innovación lingüística en cuestión de forma independiente. Sin embargo, tal consideración aparece tanto complicada como innecesaria puesto que es posible establecer vínculos históricos entre el krio y el CI de Costa Rica, a través precisamente del CI de Jamaica.

La fundación de la colonia que da origen a la población actual de criollos hablantes de krio en Sierra Leona ocurre entre 1787 y 1800. Los primeros colonos que se establecen en Freetown son 390 exesclavos y cerca de 50 prostitutas blancas procedentes de Londres; en 1791, de estos primeros colonos sobreviven solamente 48 .

En 1792, 1.100 exesclavos se establecen en Freetown, procedentes de las excolonias británicas en los Estados Unidos vía Nueva Escocia.

En 1800, 550 cimarrones procedentes de Jamaica arriban a la colonia.

En 1820 la población de Freetown está compuesta por 4.736 personas: 691 exesclavos de Estados Unidos, 487 cimarrones de Jamaica y 4.106 nativos africanos.

Entre 1808 y 1864, al abolirse la trata de esclavos por los ingleses, cerca de 84.000 esclavos liberados de los barcos esclavistas capturados por los británicos se establecen en la colonia (cf. Jones 1971; Wyse 1989: 2).

A partir de 1839 se inicia una verdadera diáspora krio (Wyse 1989: 19 y ss.) durante todo el siglo XIX y aun parte del siglo XX, la cual se extiende por gran parte de África Occidental. La influencia lingüística y cultural de los grupos de emigrantes krios, sobre todo en calidad de misioneros, comerciantes o administradores de la Corona Británica, es considerado muy importante.

La cronología de la expansión del krio por África Occidental puede resumirse de la siguiente manera: Gambia (1821), Bioko o Fernando Pó (1834), Nigeria (1840), Camerún (1845) y Ghana (1874). 
Esta cronología confirma históricamente la estrecha relación lingüística que existe entre el krio y el wescos hablado en la costa occidental de África.

Por el contrario, la hipótesis del origen americano del protocriollo inglés del Atlántico explicaría fácilmente la razón por la cual la innovación lingüística compartida por el krio y wescos, por un lado, y el CI de Guyana y el CI de Costa Rica, por otro, no afectó a las lenguas de Surinam.

Según esta hipótesis, el protocriollo inglés del Atlántico tuvo su origen en la isla de Barbados y de allí fue transportado a las diferentes regiones tanto de América como de África, según la cronología relatada anteriormente.

De acuerdo con esta hipótesis, habría que suponer simplemente que la innovación del cambio de lugar del tono alto respecto del lugar original que corresponde al acento en inglés ocurrió en una época posterior al establecimiento de la colonia de Surinam por parte de los ingleses; es decir, probablemente entre 1651 y 1667, año en que esta colonia es cedida a Holanda.

Se debe suponer también que esta innovación ocurrió presumiblemente en Barbados. Téngase en cuenta que en el (criollo) inglés de esta isla aparecen los mismos patrones de distribución tonal que se han mencionado para el CI de Guyana y para el CI de Costa Rica (cf. Holder 1972).

Esta innovación lingüística será trasportada a Jamaica en 1665 y a Guyana en 1740 directamente de Barbados. De Jamaica será llevada a Sierra Leona en 1800 y a Costa Rica en 1871. De Sierra Leona será extendida por gran parte de África Occidental desde Ghana hasta Camerún durante todo el siglo XIX.

\section{Notas}

1. El término original en inglés es English-based Atlantic Creoles.

2. Sin embargo, en su clasificación de los criollos ingleses del Atlántico, Hancock (1987: 324-5) incluye a Barbados dentro de la lista de variedades criollas.

3. Para una discusión teórica acerca de este punto véase Carter (1983).

4. Los datos para la comparación han sido tomados de las siguientes fuentes:

CI de Costa Rica: datos de trabajo de campo

CI de Guyana: Carter (1987 y 1989)

Sranan: Echteld (1961)

Saramaca: Voorhoeve (1959 y 1961) y Rountree (1972)

Djuka: Huttar \& Huttar (1972)

Krio: Fyle \& Jones (1980) y Carter (1989)

Wescos: Dwyer (1967) y Carter (1987).

5. Ejemplos tomados de Holder (1972). La transcripción fonemática de los segmentales es solo aproximada, ya que Holder presenta los ejemplos en ortografía inglesa.

6. Todos los ejemplos que aparecen a continuación son tomados de Carter (1987).

7. Todos los ejemplos son tomados de Fyle \& Jones (1980). Aunque los autores señalan también la existencia de un tono descendente, este parece no tener carácter fonémico, por lo tanto no será marcado en los ejemplos (cf. Fyle \& Jones 1980: xxiii y también Carter 1989).

8. Todos los ejemplos son tomados de Dwyer (n.d. [1967]). Por las razones expuestas el tono alto débil y el tono alto fuerte de las transcripciones de Dwyer son representados como un único tono alto. 
9. Criper-Friedman (1990: 69).

10. Ejemplos tomados de Voorhoeve (1959).

11. Todos los ejemplos son tomados de Huttar \& Huttar (1972).

12. Ejemplos tomados de Echteld (1961).

13. En las variedades tonales comparadas, los casos de palabras con más de un tono son de origen presumiblemente africano.

14. El CI de Guyana no ha sido incluido debido a que Carter (1987 y 1989) no ofrece una especificación del tono para estas palabras.

15. El símbolo (!) indica que en estas palabras no existe una seguridad absoluta sobre la asignación de los tonos.

16. Se han excluido de la comparación el wescos, el CI de Guyana, el saramaca y el djuka porque no se cuenta con material suficiente para encontrar los cognados correspondientes. Los datos que se ofrecen a continuación han sido tomados de Fyle \& Jones 1980 (krio), Echcteld 1961 (sranan) y del material de trabajo de campo (CI de Costa Rica).

17. En el CI de Costa Rica, esta palabra tiene además el significado de 'vagina'.

18. En sranan, esta palabra significa 'anciano (aged)'.

19. Echteld (1961: 83) ofrece la siguiente probable derivación histórica de esta palabra en sranan: *koko'noto > *kok'noto > ko'kronto. En el CI de Costa Rica existe también la forma / kuoknát /.

20. En sranan, la palabra es un compuesto derivado de 'fish' + 'man'.

21. Según el OED, la palabra inglesa 'macaw' proviene del arawaco 'macoya' o 'mocoya', que es una especie de palmera espinosa (Acrocomea aculeata).

22. En el CI de Costa Rica, la palabra designa al 'Hermano Araña', el embaucador, un personaje de cuentos tradicionales de origen africano.

23. En krio, la palabra significa 'pequeño'.

24. El término en sranan ha sido tomado de Taylor (1977: 159).

\section{Bibliografía}

Berry, J. 1976. Tone and intonation in Guyanese English. En: Juilland (ed.), 263-70.

Carrington, L. et al (eds.). 1983. Studies in Caribbean Language. St. Augustine: Society for Caribbean Linguistics.

Carter, H. 1983. How to be a tone language? En: Carrington et al. (eds.), 1983: 90-111.

1983. Studies in Caribbean Language. St. Augustine: Society for Caribbean Linguistics.

1987. Suprasegmentals in Guyanese: some African comparisons. En Gilbert (ed.), 213-63. 
1989. Three Creole pitch systems. En: Haik \& Tuller (eds.), 27-44

Cassidy, F. 1980. The place of Gullah. American Speech . 55: 3-15.

Criper-Friedman, L. 1990. The tone system of West African English. World Englishes . 9: 63-77.

Gilbert, G. (ed.). 1987. Pidgin and Creole languages. Honolulu: University Press of Hawaii.

Görlach, M. \& J. Holm (eds.). 1986. Focus on the Caribbean. Amsterdam: Benjamins.

Grimes, J. (ed.). 1972. Languages of the Guianas. México: Summer Institute of Linguistics.

Dwyer, D. n.d. [1967]. An introduction to West African Pidgin English. Michigan State University.

Echteld, J. 1961. The English words in Sranan. Groningen: Wolters.

Fyle, C. \& E. Jones. 1980. A Krio-English dictionary. Nueva York: Oxford University Press.

Haïk, I. \& L. Tuller (eds.). 1989. Current approaches to African linguistics 6. Dordrecht: Foris Publications.

Hancock, I. 1969. A provisional comparison of the English-based Atlantic Creoles. African Language Review . 8: 7-72.

1977. Recovering pidgin genesis. En: Valdman (ed.), 277-94.

1980. Gullah and Barbadian: origins and relationships. American Speech . 55: 17-35.

1986. The domestic hypothesis, diffusion and componentiality. En: Muysken \& Smith (eds.), 71-102.

1987. A preliminary classification of the anglophone Atlantic Creoles, with syntacticdata from thirty-three representative dialects. En: Gilbert (ed.), 264-333.

Hellinger, M. 1985. English-orientierte Pidgin- und Kreolsprachen. Darmstadt: Wissenshaftliche Buchgesellschaft.

Holder, M. 1972. Word accentual patterns in Guyanese English (GE) compared with British English (RP norm). Proceedings of the 7th International Congress of Phonetic Sciences, Montreal . 1971: 897-9. La Haya: Mouton.

Holm, J. 1986. The spread of English in the Caribbean area. En: Görlach \& Holm (eds.), 1-22.

Huttar, G \& M. Huttar. 1972. Notes on Djuka phonology. En: Grimes (ed.), 1-11.

Juilland, A. (ed.). 1976. Linguistic studies offered to Joseph Greenberg. Phonology. Saratoga: Anma Libri. 
Jones, E. 1971. Krio: an English based language of Sierra Leone. En: Spencer (ed.), 66-94.

LePage, R. \& D. DeCamp. 1960. Jamaican Creole. Londres: Macmillan.

Muysken, P. \& N. Smith (eds.). 1986. Substrata versus universals in creole genesis. Amsterdam: Benjamins.

Niles, A. 1980. Provincial English dialects and Barbadian English. Tesis doctoral: Universidad de Michigan.

Rountree, S. 1972. The phonological structure of stems in Saramaccan. En: Grimes (ed.), 1972: 22-7.

Roy, J. 1986. The structure of tense and aspect in Barbadian English Creole. En Görlach \& Holm (eds.), 141-56.

Spencer, J. (ed.). 1971. The English Language in West Africa. Londres: Longman.

Taylor, D. 1977. Languages of the West Indies. Baltimore \& Londdres: John Hopkins Univesity Press.

Valdman, A. (ed.). 1977. Pidgin and Creole linguistics. Bloomington: Indiana University Press.

Voorhoeve, J. 1959. An orthography for Saramaccan. Word. 15: 436-45.

1961. Le ton et la grammaire dans le saramaccan. Word. 17: 146-63.

1970. The regularity of sound correspondences in a Creole language (Sranan). Journal of African Languages. 9: 51-69.

Wyse, A. 1989. The Krio of Sierra Leone. Londres: Hurst. 